\section{Lithiumaugmentation zur Behandlung der bipolaren Depression}

Tom Bschor ${ }^{1}$, Ute Lewitzka' ${ }^{1}$ Michael Bauer²

1 Klinik und Poliklinik für Psychiatrie und Psychotherapie, Universitätsklinikum Carl Gustav Carus an der Technischen Universität Dresden 2 Klinik für Psychiatrie und Psychotherapie, Charité, Universitätsmedizin Berlin, Campus Charité-Mitte, Humboldt-Universität zu Berlin

psychoneuro 2003; 29 (9): 392-399

Lithiumaugmentation ist die Addition von Lithium zu einem Antidepressivum in der Akuttherapie depressiver Episoden, wenn mit dem Antidepressivum in Monotherapie keine ausreichende Wirksamkeit erzielt werden konnte. Zahlreiche doppelblinde und plazebokontrollierte Studien belegen die Wirksamkeit dieses Verfahrens bei unipolaren Depressionen. Kontrollierte Studien zur Wirksamkeit der Lithiumaugmentation bei bipolaren Depressionen existieren nicht. Aufgrund verschiedener Unterschiede, insbesondere des Risikos einer Manieinduktion durch antidepressive Behandlung, können Behandlungsstrategien der unipolaren Depression jedoch nicht ohne Weiteres auf bipolare Erkrankungen übertragen werden. Unkontrollierte Untersuchungen weisen jedoch auf eine möglicherweise sogar besonders gute Wirksamkeit bei dieser Depressionsform hin. Da Lithium eine potente antimanische Substanz ist und somit wenig verdächtig, eine Manie zu induzieren, da es darüber hinaus als Schutz vor einem Umschlag in die Manie begleitend zu einer Antidepressiva-Medikation ohnehin empfohlen wird und da es schließlich das Medikament der ersten Wahl zur Langzeitprophylaxe der bipolar-affektiven Erkrankung ist und in dieser Indikation nach erfolgreicher Lithiumaugmentation beibehalten werden kann, kann die Lithiumaugmentation auch zur Behandlung bipolarer Depressionen empfohlen werden.

L ithium wird seit Mitte des 20. Jahrhunderts systematisch in der Behandlung affektiver Erkrankungen eingesetzt und ist damit das älteste und bestuntersuchteste unter den heute verwendeten Psychopharmaka. Seine Hauptindikationen sind die Behandlung der akuten Manie und die prophylaktische Langzeittherapie rezidivierender uni- (6) und bipolarer $(2,12)$ Erkrankungen. Lithium ist unverändert das Medikament der 1. Wahl zur Phasenprophylaxe der manisch-depressiven Erkrankung (7) und in Deutschland als einziges Medika- ment für diese Indikation uneingeschränkt zugelassen. Obwohl mindestens sieben doppelblinde und randomisierte Studien in den 70erJahren auch eine akut-antidepressive Wirkung von Lithium zeigten (Übersicht: 1), setzte sich die Behandlung akuter depressiver Episoden mit einer Lithium-Monotherapie nicht durch. Dies liegt vermutlich an dem eher schwach ausgeprägten antidepressiven Effekt von Lithium und daran, dass mit den Antidepressiva unkomplizierter einsetzbare Mittel für diese Indikation zur Verfügung stehen.

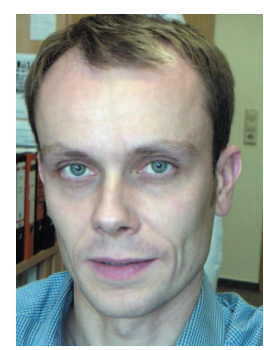

Tom Bschor

\section{Lithiumaugmentation}

Erst mit der Entdeckung der Lithiumaugmentation durch die $\mathrm{Ar}$ beitsgruppe des Kanadiers de Montigny 1981 (11) erhielt das Element auch einen festen Stellenwert in der akut-antidepressiven Behandlung. Lithiumaugmentation (lat. augmentum = Vermehrung, Zuwachs) bedeutet die Zugabe von Lithium zu einem Antidepressivum, das nach ausreichend langer Gabe (mindestens vier Wochen) in ausreichender Dosierung nicht zu einer befriedigenden Besserung der depressiven Symptomatik geführt hat (s. Abb. 1). Hierdurch kann häufig doch noch ein Ansprechen auf die antidepressive Pharmakotherapie erzielt werden $(4,9)$. Die mit der Idee der Augmentation verbundene Annahme ist nicht die einer synergistischen Kombination zweier antidepressiver Wirkstoffe, sondern die, dass das Augmentum aufgrund einer spezifischen pharmakologischen Interaktion dem Antidepressivum zur vorher nicht vorhandenen Wirkung verhilft. Im Falle der Lithiumaugmentation besteht diese theoretische (nicht bewiesene) Annahme darin, dass Lithium aufgrund seiner bekannten pro-serotonergen Eigenschaften über die durch die Antide- 


\section{Abb. 1 Prinzip der Lithiumaugmentation}

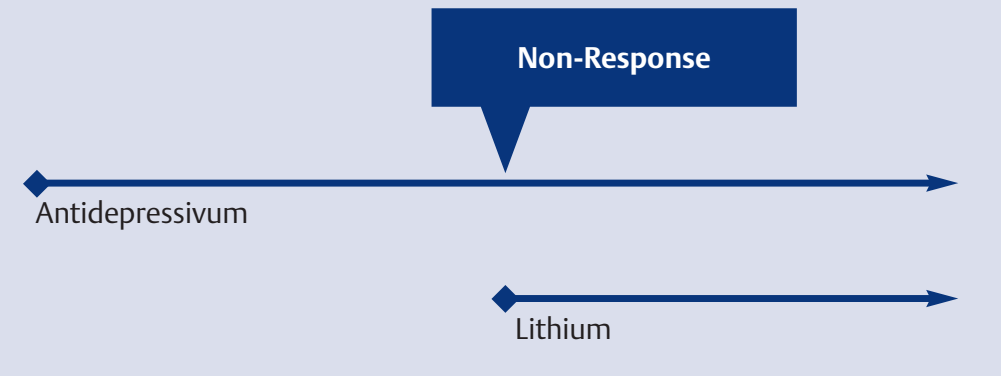

pressiva Vorbehandlung sensibilisierten Serotoninrezeptoren die positive Wirkung der Lithiumaugmentation entfaltet. Aus Tierversuchen war nämlich bekannt, dass eine regelmäßige Behandlung mit trizyklischen Antidepressiva zu einer Sensibilisierung von Serotoninrezeptoren im Vorderhirn führt.

In eine Metaanalyse zur Wirksamkeit der Lithiumaugmentation (3) konnten neun doppelblinde und randomisierte Studien eingeschlossen werden, die die Wirksamkeit einer Lithium- mit der einer Plazeboaugmentation bei - ganz überwiegend - unipolaren Depressionen verglichen hatten. Nach dieser zusammenfassenden Auswertung kann die klinische Wirksamkeit einer Lithiumaugmentation als gesichert angesehen werden (s. Abb. 2). Die Metaanalyse zeigte darüber hinaus, dass die Lithiumaugmentation mit Dosierungen, wie sie auch zur prophylaktischen Lithiumbehandlung üblich sind, durchgeführt werden, der Lithiumspiegel also im etablierten Bereich (0,6-0,9 mmol/l) liegen sollte. Sie zeigte ferner, dass die Lithiumaugmentation mit diesen Serumspiegeln für mindestens zwei Wochen durchgeführt werden sollte, bevor ihre Wirksamkeit beurteilt werden kann.

Aufgrund ihres Wesens ist die Lithiumaugmentation explizit ein Behandlungsverfahren des sogenannten „zweiten Schrittes“, also für Patienten, die auf eine Antidepressiva-Monotherapie nicht respondiert hatten. Im Vergleich zu anderen Zweitschritt-Behandlungsmethoden (Wechsel des Antidepressivum, Hochdosisbehandlung, Antidepressiva-Kombination) ist die Lithi- umaugmentation empirisch besonders gut abgesichert und daher ein, wenn nicht das Behandlungsverfahren der ersten Wahl im Falle von Nonresponse auf eine Monotherapie mit einem Antidepressivum bei unipolaren Depressionen (5).

\section{Bipolare Depression}

Depressive Episoden, die im Rahmen einer manisch-depressiven Erkrankung auftreten (bipolare Depressionen) weisen einige therapierelevante Unterschiede zu depressiven Episoden außerhalb bipolarer Erkrankungen auf, weshalb Behandlungsempfehlungen nicht ohne weiteres von den uni- auf die bipolaren Depressionen übertragen werden können. Als Hauptproblem in der Behandlung bipolarer Depressionen wird das Risiko angesehen, durch eine antidepressive Medikation ein
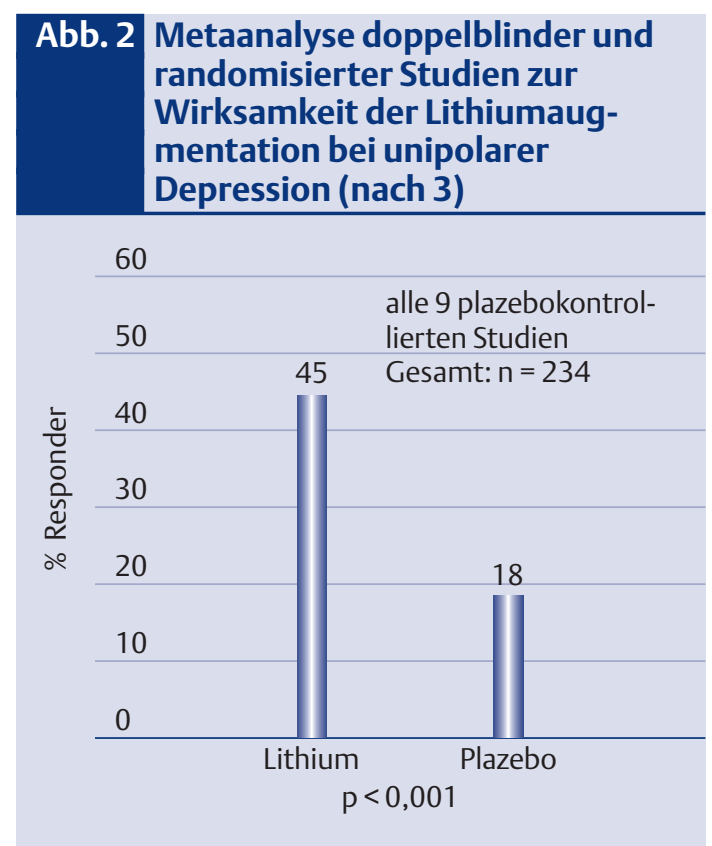
Umschlagen (switch) in eine Manie auszulösen. Darüber hinaus wurde beobachtet, dass eine antidepressive Behandlung zu einer zunehmenden Verkürzung der Zyklusdauer (Zeitspanne vom Beginn einer affektiven Phase bis zum Beginn der nächsten affektiven Phase) führen kann. In der Erhaltungstherapie (6-12 Monate nach Remission einer depressiven Episode), in der aufgrund des erhöhten Rezidiv-Risikos für unipolare Depressionen eindeutig eine unveränderte Fortführung der zur Remission führenden Medikation empfohlen wird, besteht bei bipolaren Depressionen das therapeutische Dilemma zwischen dem Risiko, durch das Fortführen der antidepressiven Medikation eine Manie zu induzieren, und dem Risiko, durch ein rasches Absetzen einen depressiven Rückfall zuzulassen.

Aufgrund der genannten Risiken einer Behandlung mit Antidepressiva wird daher von einigen Autoren empfohlen, eine bipolare Depression (zunächst) nur mit einem Medikament aus der Gruppe der Stimmungsstabilisierer, das, wie z.B. Lithium, auch antidepressive Eigenschaften aufweist, zu behandeln (15). Andere Autoren favorisieren von Beginn an eine AntidepressivaMedikation, die aber nicht ohne begleitenden antimanischen Schutz mit einem Stimmungsstabilisierer durchgeführt werden sollte (10).

\section{Lithiumaugmentation bei bipolarer Depression}

Eine Lithiumaugmentation kommt bei bipolar depressiven Patienten nur in Betracht, wenn sie nicht ohnehin bereits Lithium in phasenprophylaktischer Indikation erhalten. Aufgrund theoretischer Erwägungen ist eine Lithiumaugmentation aber für den Fall, dass eine antidepressive Medikation in der Behandlung einer bipolaren Depression nicht zur Response führte, aus verschiedenen Gründen attraktiv:

Durch die Addition von Lithium zu einem bislang nicht wirksamen Antidepressivum führt man nicht nur eine Lithiumaugmentation durch, sondern addiert zugleich ein Medikament, das einen wirkungsvollen (wenngleich natürlich nicht vollständigen) Schutz vor einem Antidepressiva-induzierten Umschlagen in eine Manie bietet (8). Darüber hinaus ist Lithium als wirkungsvoll antimanisches Medikament wenig verdächtigt, seinerseits manieauslösend zu wirken. Schließlich kann Lithium, insbesondere wenn die Lithiumaugmentation eine gute antidepressive Wirkung entfaltet, dem Patienten anschließend zur langfristigen Prophylaxe der bipolar affektiven Erkrankung empfohlen werden.

Die wissenschaftlich-empirischen Belege für die Wirksamkeit einer Lithiumaugmentation bei bipolaren Depressionen sind jedoch dürftig: Unter den 234 Patienten aus neun plazebokontrollierten, doppelblinden Studien der oben bereits erwähnten Metaanalyse (3) befanden sich lediglich 14 Patienten mit bipolarer Depression. Eine spezifische Aussage für diese Indikation ist daher aus dieser Arbeit nicht ableitbar. Zwei nicht-kontrollierte Studien weisen jedoch in Richtung einer möglicherweise sogar besonders guten Wirksamkeit der Lithiumaugmentation bei bipolaren Depressionen: Rybakowski und Matkowski (14) fanden bei den 14 bipolar depressiven Patienten unter den 51 untersuchten Patienten ein signifikant häufigeres Ansprechen auf die Lithiumaugmentation (in 79\%) als bei den unipolar depressiven Patienten (46\%). In einer retrospektiven Untersuchung sprachen bipolar Depressive ebenfalls häufiger auf die Lithiumaugmentation an (13). Beide Studien machen keine Angaben zu einer möglichen Manie-Induktion unter der Lithiumaugmentation.

Prospektive, randomisierte und plazebokontrollierte Studien zur Wirksamkeit der Lithiumaugmentation bei bipolaren Depressionen sind also wünschenswert. Bis diese vorliegen kann eine Lithiumaugmentation aber aufgrund der Datenlage und der theoretischen Überlegungen auch für bipolar depressive Patienten empfohlen werden. Wenn auch die Wirksamkeit der Lithiumaugmentation bei bipolarer Depression nicht empirisch abgesichert ist, so wird doch mit Lithium ein Medikament addiert, das bei manisch-depressiver Erkrankung in zwei anderen Indikationen (als Monotherapeutikum zur Behandlung bipolarer Depressionen und als Phasenprophylaktikum) aufgrund kontrollierter, randomisierter Studien klar empfohlen werden kann.

\section{Lithium Augmentation in the Treat-} ment of Bipolar Depression

Lithium augmentation is the addition of lithium to an ongoing medication with an antidepressant in the treatment of acute depression in case that the antidepressant alone has failed. Several double-blind, placebo-controlled studies demonstrate the efficacy of this treatment strategy in unipolar depression. However, there are no controlled studies on the efficacy of lithium augmentation in bipolar depression, and for some particular differences between unipolar and bipolar depression, especially for the risk of a treatment-induced switch into mania, treatment strategies may not be transferred from unipolar to bipolar depression that easily. However, uncontrolled studies suggest a perhaps especially fine efficacy in bipolar depression. Because of its strong antimanic properties, lithium (augmentation) is unlikely to induce a switch into mania. Furthermore, guidelines anyway recommend to add lithium to an antidepressant in the treatment of acute bipolar depression to reduce the risk of an antidepressant-induced switch into mania. Third, for lithium is the firstline prophylactic treatment in bipolar disorder, it can be continued for longterm treatment after a successful lithium augmentation. Taken all together, despite missing controlled studies on lithium augmentation in bipolar depression, lithium augmentation can be recommended also for this type of depression.

\section{Key Words:}

lithium augmentation - bipolar depression - antidepressant - switch into mania - refractory depression

\section{Literatur}

1. Adli M, Bschor T, Canata B, Döpfmer S, Bauer M. Lithium in der Behandlung der akuten Depression. Fortschr Neurol Psychiat 1998; 66: 435-441 
2. American Psychiatric Association. Practice guideline for the treatment of patients with bipolar disorder (second edition). American Psychiatric Publishing, Inc., Washington, DC, 2002

3. Bauer M, Döpfmer S. Lithium augmentation in treatment-resistant depression - A meta-analysis of placebo-controlled studies. J Clin Psychopharmacol 1999; 19: 427434

4. Bauer M, Forsthoff A, Baethge C, Adli M, Berghöfer A, Döpfmer S, Bschor T. Lithium augmentation therapy in refractory depression - Update 2002. Eur Arch Psychiatry Clin Neurosci 2003; 253: 132-139

5. Bauer M, Whybrow PC, Angst J, Versiani M, Möller HJ. World Federation of Societies of Biological Psychiatry (WFSBP) Guidelines for biological treatment of unipolar depressive disorders, Part 1: Acute and continuation treatment of major depressive disorder. World J Biol Psychiatr 2002; 3: 5-43

6. Bauer M, Whybrow PC, Angst J, Versiani M, Möller HJ. World Federation of Societies of Biological Psychiatry (WFSBP) Guidelines for biological treatment of unipolar depressive disorders, Part 2: Maintenance Treatment of Major Depressive Disorder \& Treatment of Chronic Depressive Disorders and Subthreshold Depressions. World J Biol Psychiatr 2002; 3: 69-86
7. Berghöfer A, Adli M, Baethge C, Bauer M, Bschor T, Müller-Oerlinghausen B, Schäfer M, Willich S. Phasenprophylaxe. In: Deutsche Gesellschaft für Bipolare Störungen e. V. (Hg.) Weißbuch Bipolare Störungen in Deutschland. Hamburg, ConferencePoint Verlag, 2003: S. 131-200

8. Bottlender R, Rudolf D, Strauss A, Möller $\mathrm{H}$. Mood-stabilisers reduce the risk of developing antidepressant-induced maniform states in acute treatment of bipolar I depressed patients. J Affect Disord 2001; 63: 79-83

9. Bschor T, Canata B, Müller-Oerlinghausen B, Bauer M. Predictors of response to lithium augmentation in tricyclic antidepressant-resistant depression. J Affect Disorders $2001 ; 64: 261-265$

10. Möller HJ, Grunze H. Have some guidelines for the treatment of acute bipolar depression gone too far in the restriction of antidepressants? Eur Arch Psychiatry Clin Neurosci 2000; 250: 57-68

11. Montigny de C, Grunberg F, Mayer A Deschenes JP. Lithium induces rapid relief of depression in tricyclic antidepressant drug non-responders. Br J Psychiatry 1981; 138: 252-256

12. Müller-Oerlinghausen $B$, Berghöfer $A$, Bauer M. Bipolar disorder. Lancet 2002; 359: 241-247
13. Nelson JC, Mazure CM. Lithium augmentation in psychotic depression refractory to combined drug treatment. Am J Psychiatry 1986; 143: 363-366

14. Rybakowski J, Matkowski K. Adding lithium to antidepressant therapy: factors related to therapeutic potentiation. Eur Neuropsychopharmacol 1992; 2: 161-165

15. Sachs GS. Bipolar mood disorder: practical strategies for acute and maintenance phase treatment. J Clin Psychopharmacol 1996; 16 (Suppl 1): 32-47

\section{Korrespondenzadresse:}

Dr. med. habil. Tom Bschor

Klinik und Poliklinik für Psychiatrie und Psychotherapie

Universitätsklinikum Carl Gustav Carus

Technische Universität Dresden

Fetscherstraße 74

D-01307 Dresden

bschor@mailbox.tu-dresden.de 